\title{
Mass, velocity anisotropy, and pseudo phase-space density profiles of Abell 2142
}

\author{
E. Munari ${ }^{1}$, A. Biviano ${ }^{2,3}$, and G. A. Mamon $^{3}$ \\ 1 Astronomy Unit, Department of Physics, University of Trieste, via Tiepolo 11, 34131 Trieste, Italy \\ e-mail: munari@oats.inaf.it \\ 2 INAF/Osservatorio Astronomico di Trieste, via Tiepolo 11, 34131 Trieste, Italy \\ e-mail: biviano@oats.inaf.it \\ 3 Institut d'Astrophysique de Paris (UMR 7095: CNRS \& UPMC), 98 bis Bd Arago, 75014 Paris, France \\ e-mail: gam@iap.fr
}

Received 6 August 2013 / Accepted 12 April 2014

\begin{abstract}
Aims. We aim to compute the mass and velocity anisotropy profiles of Abell 2142 and, from there, the pseudo phase-space density profile $Q(r)$ and the density slope - velocity anisotropy $\beta-\gamma$ relation, and then to compare them with theoretical expectations. Methods. The mass profiles were obtained by using three techniques based on member galaxy kinematics, namely the caustic method, the method of dispersion-kurtosis, and MAMPOSSt. Through the inversion of the Jeans equation, it was possible to compute the velocity anisotropy profiles.

Results. The mass profiles, as well as the virial values of mass and radius, computed with the different techniques agree with one another and with the estimates coming from X-ray and weak lensing studies. A combined mass profile is obtained by averaging the lensing, X-ray, and kinematics determinations. The cluster mass profile is well fitted by an NFW profile with $c=4.0 \pm 0.5$. The population of red and blue galaxies appear to have a different velocity anisotropy configuration, since red galaxies are almost isotropic, while blue galaxies are radially anisotropic, with a weak dependence on radius. The $Q(r)$ profile for the red galaxy population agrees with the theoretical results found in cosmological simulations, suggesting that any bias, relative to the dark matter particles, in velocity dispersion of the red component is independent of radius. The $\beta-\gamma$ relation for red galaxies matches the theoretical relation only in the inner region. The deviations might be due to the use of galaxies as tracers of the gravitational potential, unlike the non-collisional tracer used in the theoretical relation.
\end{abstract}

Key words. methods: observational - galaxies: clusters: general - galaxies: kinematics and dynamics - galaxies: general

\section{Introduction}

The measure of the mass of cosmological objects, such as clusters of galaxies, has proven to be an important tool for cosmological applications. The mass is not a direct observable, and many techniques have been developed to infer it by measuring observable quantities. Two methods that are widely used to infer the mass profile of galaxy clusters are the X-ray and the lensing techniques. The former makes use of the observations of the $\mathrm{X}$-ray emission of the hot intracluster plasma (ICM). The lensing technique makes use of the relativistic effect of distortion of the trajectories of light emitted by distant background galaxies caused by the mass of the observed cluster. These two methods have some limitations either way. In the case of X-ray technique, the limitation comes from the usual assumption that the plasma of the cluster is in hydrostatic equilibrium, and the cluster approximately spherically symmetric (Ettori et al. 2002) with no important recent merger activity (Böhringer \& Werner 2010). As for the lensing technique, its limitation is that it only allows computing the projected mass, and this includes all the line-ofsight (LOS) mass contributions. The complementarity of the different techniques is a strong advantage for reliably constraining the mass of a cluster.

In this article, we use another kind of information that comes from the kinematics of the galaxies belonging to the observed cluster. In fact, the potential well of the cluster, due to the mass, is the main driver of the orbital motion of the galaxies, which in the absence of mutual interactions, can be treated as test particles in the gravitational potential of the cluster. The kinematics of galaxies therefore carries the information about the mass content of the cluster. The motion takes place in a six-dimensional phase space, but the observations are able to capture only three of these dimensions, namely two for the position and one for the LOS velocity. This is one of the most important limitations of a mass estimate via observation of the kinematics of galaxies. To overcome this problem, most methods assume spherical symmetry.

A spherically symmetric density profile following the universal relation provided by Navarro et al. (1996; 1997; NFW hereafter) has often been adopted in these analyses. Such a profile is characterized by its "scale radius" parameter, which is the radius where the logarithmic slope of the density profile is equal to -2 . With the advent of simulations with increasingly higher resolution, the universality of the NFW density profile has been questioned (see e.g. Navarro et al. 2004; Vogelsberger et al. 2011; Ludlow et al. 2013). While the self-similarity of the density profiles of DM-only haloes may not hold as well as initially thought, another physical parameter appears to have a quasiuniversal radial profile, the pseudo phase-space density (PPSD) $Q(r)=\rho / \sigma^{3}$, where $\rho$ is the total matter density profile and $\sigma$ the 
3D velocity dispersion of the tracers of the gravitational potential (Taylor \& Navarro 2001; Ludlow et al. 2010). Still, some doubts have been raised about its universality (Ludlow et al. 2011). The use of the radial velocity dispersion instead of the total one has proven to be a valid and robust alternative for computing the PPSD, in this case called $Q_{r}(r)$. The link between these two formulations of the PPSD is constrained by the velocity anisotropy (hereafter, anisotropy) of the system, which plays a non trivial role in shaping the structure of a system. The density profile and the anisotropy profile are in fact found to correlate. An empirical relation is provided by Hansen \& Moore (2006) and Ludlow et al. (2011), linking the logarithmic slope of the density profile $\gamma=\mathrm{d} \ln \rho / \mathrm{d} \ln r$ and the anisotropy $\beta(r)=1-\left(\sigma_{\mathrm{t}} / \sigma_{r}\right)^{2}$, where $\sigma_{r}$ and $\sigma_{\mathrm{t}}$ are the velocity dispersions of the radial component and of one of the two tangential components, respectively. Hereafter we refer to anisotropy as $\beta$ or the equivalent $\sigma_{r} / \sigma_{\mathrm{t}}=1 / \sqrt{1-\beta^{2}}$. We also denote the relation between anisotropy and logarithmic slope of the density profile as the $\beta-\gamma$ relation.

In this article, we study Abell 2142 (A2142 hereafter), a rich galaxy cluster at $z \sim 0.09$. The large number of galaxy members allows us to derive the total mass profile, to test different models, as well as to perform dynamical analyses in order to derive the anisotropy of the orbits of galaxies that allows to compute the pseudo phase-space density profile and the $\beta-\gamma$ relation. This cluster shows evidence of some recent mergers. In fact, the X-ray emission appears to have an elliptical morphology elongated in the north-west south-east direction (Markevitch et al. 2000; Akamatsu et al. 2011). The merging scenario is also supported by the presence of substructures of galaxies lying along the direction of the cluster elongation, as found in the SZ maps by Umetsu et al. (2009), lensing analysis by Okabe \& Umetsu (2008), and analysis of the distribution of LOS velocities of Owers et al. (2011). However, after analysing XMMNewton images to investigate the cold fronts of A2142, Rossetti et al. (2013) argue that the mergers have intermediate mass ratios rather than major ones.

Throughout this paper, we adopt a $\Lambda C D M$ cosmology with $H_{0}=70 \mathrm{~km} \mathrm{~s}^{-1} \mathrm{Mpc}^{-1}, \Omega_{0}=0.3, \Omega_{\Lambda}=0.7$. The virial quantities are computed at radius $r_{200}{ }^{1}$.

\section{The data}

The photometric information has been obtained from the SDSS DR7 database ${ }^{2}$ after searching for the galaxies that have $238^{\circ} .983<\mathrm{RA}<240^{\circ} .183,26^{\circ} .633<$ Dec $<27^{\circ} .834$ and petroMag $_{r^{\prime}}<22$. The spectroscopic information has been provided by Owers et al. (2011). The full sample is composed of 1631 galaxies with both photometric and spectroscopic information. The cluster centre is assumed to coincide with the X-ray centre provided by De Grandi \& Molendi (2002).

Two algorithms have been used to select cluster members, those of den Hartog \& Katgert (1996) and of Mamon et al. (2013), hereafter dHK and clean, respectively. Both identify cluster members on the basis of their location in projected phasespace $^{3}: R, v_{\text {rest }}$, using the spectroscopic values for the velocities.

\footnotetext{
${ }^{1} r_{\Delta}$ is the radius within which the mean density is $\Delta$ times the critical density of the Universe.

2 http://cas.sdss.org/astro/en/tools/chart/chart.asp

$3 R$ is the projected radial distance from the cluster centre. We assume spherical symmetry in the dynamical analyses. The rest-frame velocity is defined as $v=c(z-\bar{z}) /(1+\bar{z})$. The mean cluster redshift $\bar{z}$ is re defined at each new iteration of the membership selection, until convergence.
}

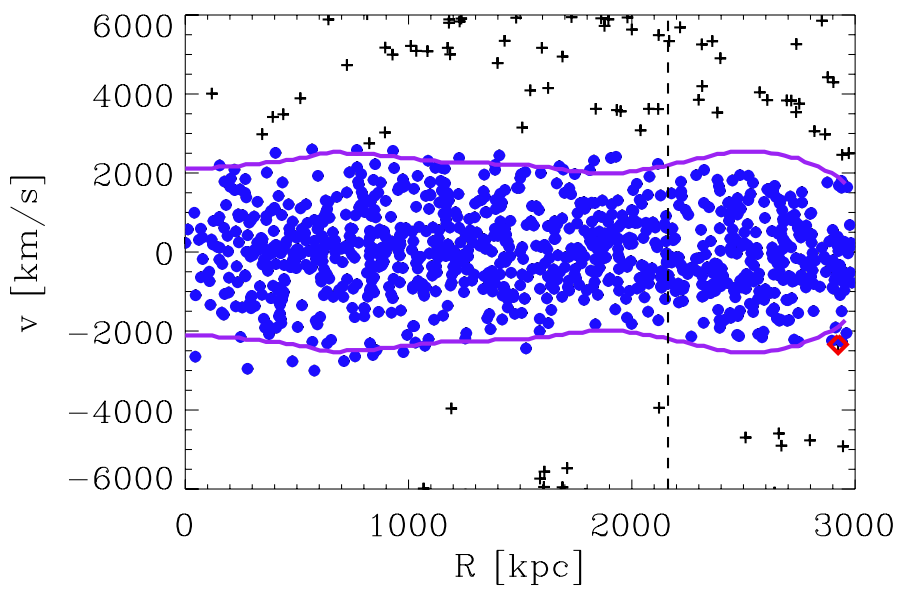

Fig. 1. Distribution of the galaxies of Abell 2142 in the projected phasespace of projected radii and LOS rest-frame velocities. Cluster members, as identified by both dHK and clean algorithms, are denoted by blue filled dots. The red diamond is the galaxy identified as member by $\mathrm{dHK}$ but not by the clean algorithm. The purple solid lines are the caustic, described in Sect. 3. The vertical dashed line locates the virial radius of the combined model (see Sect. 4).

We adopt the membership determination of $\mathrm{dHK}$, resulting in 996 members. In fact, the clean algorithm removes one more galaxy that is very close to the distribution of selected members and therefore seems unlikely to be an interloper. Anyway, this galaxy is $\approx 3 \mathrm{Mpc}$ from the cluster centre, which should make no difference in the analysis here. Figure 1 shows the location of galaxies in projected phase-space with the identification of cluster member galaxies using the two methods. We use the method described in Appendix B of Mamon et al. (2013) to obtain a preliminary estimate of the virial radius from the velocity dispersion of the cluster members. The value we obtain is $2.33 \mathrm{Mpc}$. This is used later as an initial-guess value for the virial radius, only to be successively refined with more sophisticated techniques (see Sect. 4.2).

The cluster mean redshift and LOS velocity dispersion, as well as their uncertainties, have been computed using the biweight estimator (Beers et al. 1990) on the redshifts and restframe velocities of the members: $\langle z\rangle=0.08999 \pm 0.00013$, $\sigma_{\text {los }}=1193_{-61}^{+58} \mathrm{~km} \mathrm{~s}^{-1}$.

\subsection{The colour identification}

We identify the red sequence iteratively by fitting the $g^{\prime}-r^{\prime}$ vs. $r^{\prime}$ colour-magnitude relation of galaxies with $r^{\prime}<19.5$ and $g^{\prime}-r^{\prime}>0.7$, then selecting galaxies within $\pm 2 \sigma$ of the found sequence (where $\sigma$ is the dispersion around the best fit relation). We refer to the cluster members within $\pm 2 \sigma$ of the red sequence, and those above this range, as red sequece galaxies, and to the cluster members more than $2 \sigma$ below the red sequence as blue galaxies, as shown in Fig. 2.

\subsection{Removal of substructures}

Owers et al. (2011) found some substructures in A2142, probably groups that have been recently accreted by the cluster. These substructures can alter the kinematics of the system since they still retain memory of the infall kinematics. For this reason, we compute the mass profile of the system excluding the galaxies belonging to these substructures. In particular we consider the largest substructures in this cluster, namely S2, S3, and S6, 


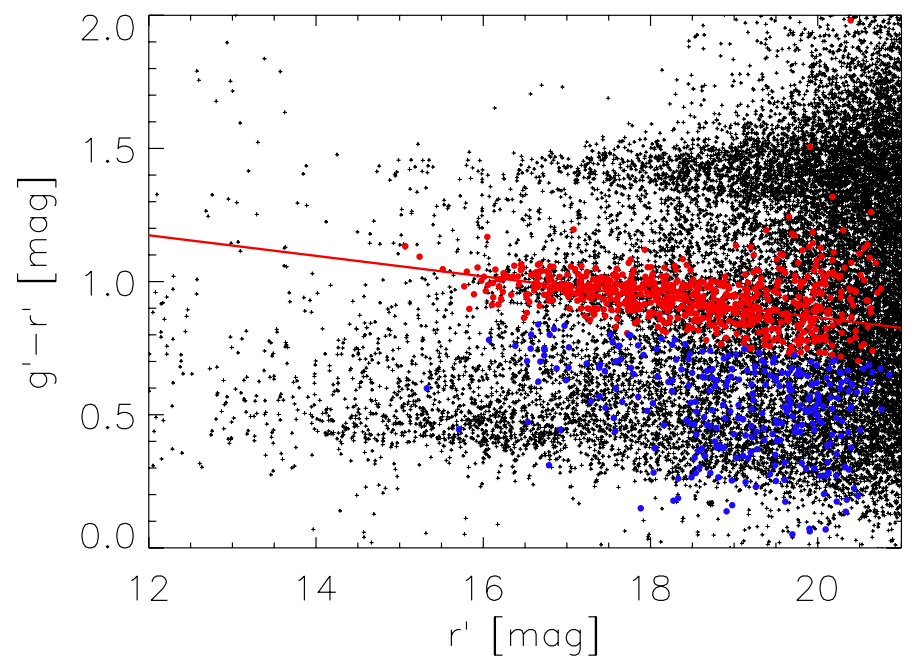

Fig. 2. Colour magnitude diagram $g^{\prime}-r^{\prime}$ vs. $r^{\prime}$. Red (blue) points are relative to red (blue) member galaxies. Black points are galaxies for which we have photometric information, that are not identified as members. The red solid line locates the red sequence.

Table 1. Coordinates with respect to the cluster center, radii, and number of galaxies of the three main substructures, as found by Owers et al. (2011).

\begin{tabular}{ccccc}
\hline \hline & $x_{\mathrm{c}}[\mathrm{Mpc}]$ & $y_{\mathrm{c}}[\mathrm{Mpc}]$ & $r[\mathrm{Mpc}]$ & $N_{\mathrm{gal}}$ \\
\hline S2 & 0.600 & 0.763 & 0.467 & 49 \\
S3 & 2.007 & 1.567 & 0.700 & 54 \\
S6 & 2.327 & -0.180 & 0.812 & 53 \\
\hline
\end{tabular}

following the nomenclature of Owers et al. (2011). Therefore, we remove galaxies inside circles, the centres and radii of which are reported in Table 1.

\subsection{The samples}

Some of the techniques (described in Sect. 3) that we use to compute the mass profile of the cluster rely upon the assumption of equilibrium of the galaxy population. Red galaxies are more likely an older cluster population than blue galaxies, probably closer to dynamical equilibrium (e.g. Moss \& Dickens 1977; van der Marel et al. 2000). For this reason, red galaxies constitute a better sample for such techniques. Among red galaxies, those outside substructures (see Sect. 2.2) are the most likely to be in dynamical equilibrium. We therefore use these galaxies for determining the mass profile.

The three samples that are used hereafter are as follows. We refer to the sample made of all the member galaxies to as the ALL sample. BLUE is the sample made of blue galaxies, and RED is the sample made of red galaxies not belonging to the substructures described in Sect. 2.2. See Table 2 for a summary of the number of galaxies belonging to each sample. The ALL and BLUE samples do contain substructures.

\section{The techniques}

In this section, we briefly describe the main features of the three different techniques used in this work to compute the mass profile of A2142. Besides the virial values of radius and mass, we obtain estimates of the mass scale radius, which is where the logarithmic slope of the total density profile is equal to -2 , from
Table 2. Number of galaxies in the three samples.

\begin{tabular}{lcc}
\hline \hline Sample & $n_{\text {tot }}$ & $n_{200}$ \\
\hline ALL & 996 & 706 \\
RED & 564 & 447 \\
BLUE & 278 & 162 \\
\hline
\end{tabular}

Notes. For each sample, the total number of member galaxies and the number of member galaxies within $r_{200}$ are shown, the latter being the value of the combined model (see Sect. 4).

which it is possible to recover the cluster mass profile. These methods all assume spherical symmetry.

\subsection{Methods}

DK: the dispersion kurtosis technique, hereafter shortened to DK, first introduced by Łokas (2002), relies upon the joint fit of the LOS velocity dispersion and kurtosis profiles of the cluster galaxies. In fact, fitting only the LOS velocity dispersion profile to the theoretical relation coming from the projection (see Mamon \& Łokas 2005b for single integral formulae for the case of simple anisotropy profiles) of the Jeans (1904) equation (see e.g. Binney \& Mamon 1982; Binney $\&$ Tremaine 1987) does not lift the intrinsic degeneracy between mass profile and anisotropy profile determinations (as Łokas \& Mamon 2003 showed for the Coma cluster). This technique assumes dynamical equilibrium of the system, and it allows us to estimate the virial mass, the mass scale radius and the value of the cluster velocity anisotropy, considered as a constant with radius ${ }^{4}$.

MAMPOSSt: the MAMPOSSt technique, recently developed by Mamon et al. (2013), performs a maximum likelihood fit of the distribution of galaxies in projected phase space, assuming models for the mass profile, the anisotropy profile, the projected number density profile and the 3D velocity distribution. In particular, for our analysis we used different NFW models for the mass and the projected number density profiles, either a simplified Tiret profile (Tiret et al. 2007) or a constant value for the anisotropy profile and a Gaussian profile for the 3D velocity distribution. As in the DK method, MAMPOSSt assumes dynamical equilibrium of the system. By this method we estimate the virial mass, the scale radius of the mass density profile, and the value of anisotropy of the tracers.

Caustic: the caustic technique, introduced by Diaferio \& Geller (1997), is different from the other two methods because it does not require dynamical equilibrium. As a result, this technique also provides the mass distribution beyond the virial radius. In projected phase space (see Fig. 1), member galaxies tend to lie in a region around $v_{\mathrm{los}}=$ $0 \mathrm{~km} \mathrm{~s}^{-1}$. Measuring the velocity amplitude $\mathcal{A}$ of the galaxy distribution gives information about the escape velocity of the system. In turn, the escape velocity is related to the potential, hence the mass profile: $M(r)=M\left(r_{0}\right)+$ $(1 / G) \int_{r_{0}}^{r} \mathcal{A}^{2}(s) \mathcal{F}_{\beta}(s) \mathrm{d} s$, where $\mathcal{F}_{\beta}(r)=-2 \pi G(3-2 \beta) /(1-$ $\beta) r^{2} \rho(r) / \Phi(r)$ (Diaferio 1999). Because $\mathcal{F}_{\beta}$ is usually approximated with a constant value (Diaferio 1999; Serra et al. 2011), it is customary to call it a "parameter".

4 Richardson \& Fairbairn (2013) have recently extended the DK method to more general anisotropy profiles. 
Since the DK and MAMPOSSt techniques make use of the assumption of dynamical equilibrium of the system, the use of the RED sample allows a more correct application of those techniques, since this sample is likely to be the most relaxed sample. In fact these methods just need a tracer that obeys the Jeans equation. As long as we consider a collisionless tracer, spherical symmetry, no streaming motions, and a stationary system, DK and MAMPOSSt are able to reliably recover the mass content of the cluster. On the other hand, we use the ALL sample for the caustic technique.

As discussed in Sect. 1, some studies suggest an elliptical morphology of the system, with evidence of some recent intermediate mass-ratio mergers. Although this might violate the assumptions of both spherical symmetry and equilibrium required in DK and MAMPOSSt, and the spherical symmetry alone for the Caustic technique, these methods are not strongly affected by this. In fact, they have been tested on $\triangle$ CDM haloes extracted from simulations. Although these haloes are neither spherical nor fully relaxed, and they present substructures, the DK (Sanchis et al. 2004), MAMPOSSt (Mamon et al. 2013) and Caustic (Serra \& Diaferio 2013; Gifford et al. 2013) techniques provide reliable estimates of halo masses. As we see below (Sect. 4), the fairly close results of these dynamical methods with those from the weak lensing analysis (which does not assume equilibrium) of Umetsu et al. (2009) suggest that this cluster cannot be far from dynamical equilibrium.

In all three methods, we consider the scale radius of the galaxy distribution and the scale radius of the mass distribution as two separate and independent parameters.

\subsection{Practical implementation}

To compute the parameter values with the MAMPOSSt technique, we have considered the galaxies of RED sample within the "first guess" virial radius, presented in Sect. 2. As discussed in Mamon et al. (2013; in particular see their Table 2), MAMPOSSt does not critically depend on this choice ${ }^{5}$. We then performed a Markov chain Monte Carlo (MCMC) procedure (see e.g. Lewis \& Bridle 2002), using the public CosmoMC code of A. Lewis ${ }^{6}$. In MCMC, the parameter space is sampled following a procedure that compares the posterior (likelihood times prior) of a point in this space with that of the previous point, and decides whether to accept the new point following a criterion that depends on the two posteriors. We use the MetropolisHastings algorithm. The next point is chosen at random from a hyperellipsoidal Gaussian distribution centred on the current point. This procedure ensures that the final density of points in the parameter space is proportional to the posterior probability. MCMC then returns probability distributions as a function of a single parameter, or for several parameters together. Here, the errors on a single parameter are computed by marginalizing the posterior probabilities over the other two free parameters.

For the caustic technique, we use the ALL sample, since the equilibrium of the sample is not required, also considering the galaxies beyond the virial radius. To apply the caustic technique, the $\mathcal{F}_{\beta}$ parameter (Diaferio 1999) must be chosen. The choice of the parameter is quite arbitrary, so we tested three different choices: the constant value 0.5 , as first suggested in Diaferio (1999); the constant value 0.7 as suggested in Serra et al. (2011);

\footnotetext{
5 However, beyond $\approx 2.5 r_{200}$, the infall streaming motions are important enough that the usual Jeans equation is inadequate for determining the radial velocity dispersion (Falco et al. 2013).

6 http://cosmologist.info/cosmomc
}

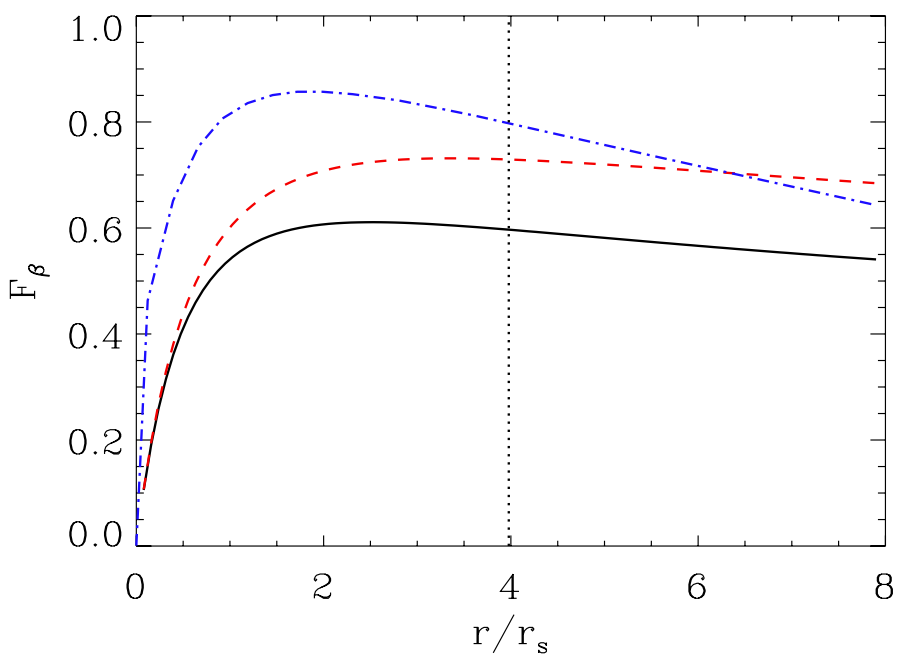

Fig. 3. $\mathcal{F}_{\beta}$ parameter as a function of clustercentric distance for an NFW model. Black solid line refers to the isotropic case, while red dashed line refers to an ML anisotropy (Mamon \& Łokas 2005b) with $r_{\text {anis }}=r_{s}$. Blue dash dotted line refers to the $\mathcal{F}_{\beta}$ by Biviano \& Girardi (2003). The dotted vertical line locates the virial radius of the combined model (see Sect. 4).

and the profile described in Biviano \& Girardi (2003). The last is a smooth approximation of the $\mathcal{F}_{\beta}(r)$ derived from numerical simulations by Diaferio (1999). The actual values of $\mathcal{F}_{\beta}$ are not likely to be very different from these we decided to test. In fact, Fig. 3 shows that an NFW model leads to $\mathcal{F}_{\beta}=0.6$ at $r=4 r_{s} \simeq r_{200}$ for isotropic orbits, while for orbits with ML (Mamon \& Łokas 2005a) anisotropy, it produces $\mathcal{F}_{\beta}=0.7$ at $r=4 r_{s} \simeq r_{200}$. As a comparison, in Fig. 3 the profile by Biviano \& Girardi (2003) is shown. It has higher values in the centre, but rapidly falls in the outer regions. The value 0.5 allows us to take both the innermost region, where the values of $\mathcal{F}_{\beta}$ are very low, and the outer part, where the values are larger and closer to 0.7 into account.

When using $\mathcal{F}_{\beta}=0.7$ and the anisotropy profile of Biviano \& Girardi (2003), the estimated virial masses are much greater than those obtained with the other techniques that rely on the dynamics of galaxies, as well as the results coming from the X-ray and the weak lensing analysis (see below). Therefore we decided to consider only the caustic technique with $\mathcal{F}_{\beta}=0.5$ (the same value has been recently adopted by Geller et al. 2013). Given that for $r_{s}<r<4 r_{s} \approx r_{200}$ one can approximate $\mathcal{F}_{\beta} \simeq$ cst typically to $\pm 11 \%$ accuracy, the mass profile returned by the caustic method changes normalization but not the shape for different values of $\mathcal{F}_{\beta}$. Therefore this method turns out to be very useful for constraining the mass profile shape, since it does not assume a parametric profile like an NFW, so that we can check whether the assumption of NFW for the mass profile is a good one. We adopt $r_{0}=0$, which relieves us from the choice of a mass at some finite radius $r_{0}$. Once we have computed the mass profile, we fit it with an NFW profile to obtain an estimate of the mass scale radius.

\subsection{The scale radius of galaxy distribution}

The NFW scale radius of the galaxy distribution is used as input for the DK and MAMPOSSt analyses, therefore it has been computed for the RED sample. The number density profile of the spectroscopic sample is affected by the incompleteness issue. We need to known the distribution of tracers along the LOS. 
E. Munari et al.: Mass, velocity anisotropy, and pseudo phase-space density profiles of Abell 2142

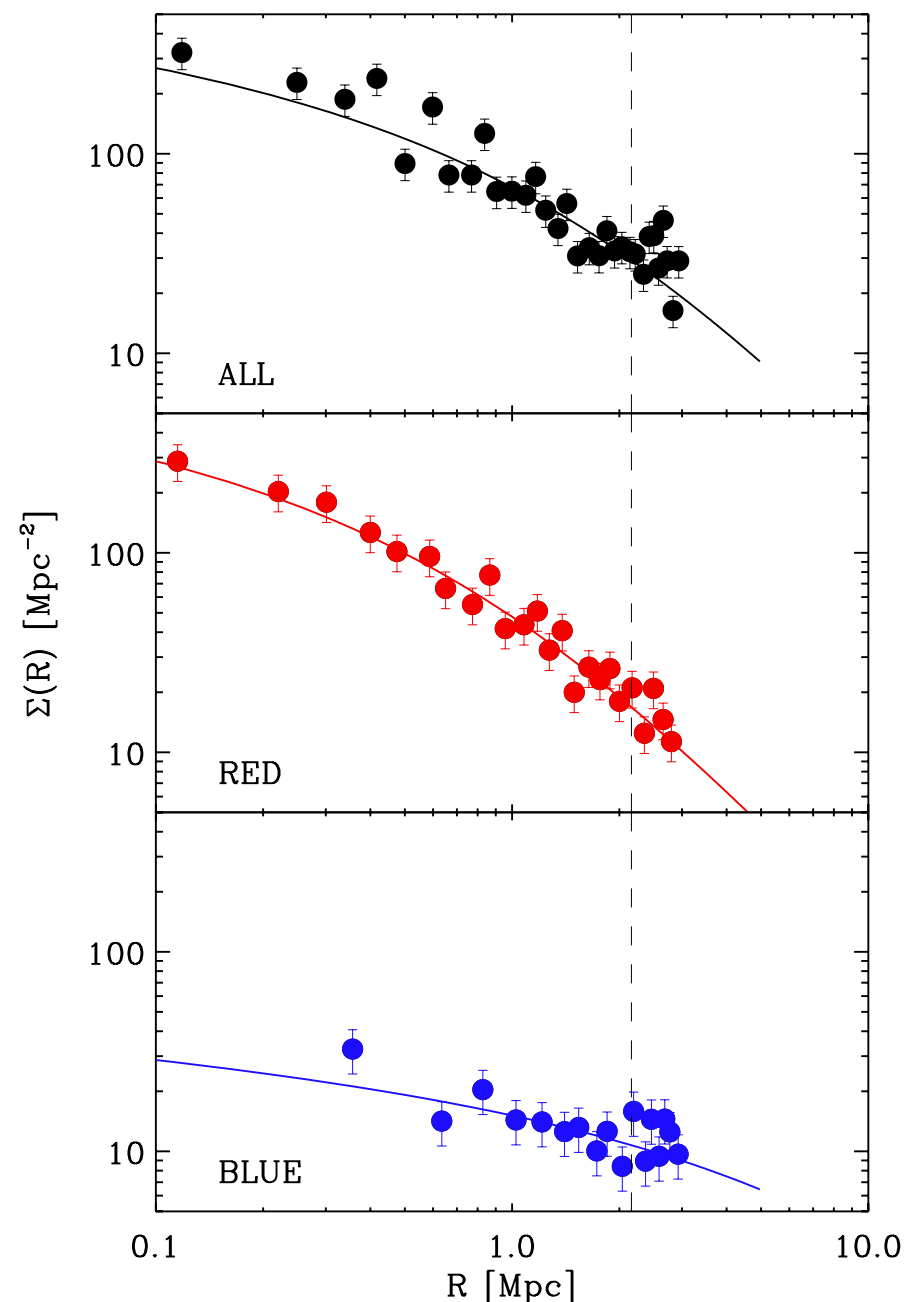

Fig. 4. Surface number density profiles for the ALL, RED, and BLUE samples, along with their best-fit projected NFW profiles. The dashed vertical line locates the virial radius of the combined model (see Sect. 4).

Assuming spherical symmetry, we can adopt the deprojection of the tracer surface density profile, but we must first correct for spectroscopic incompleteness. Owers et al. (2011, see their Fig. 2) have measured their spectroscopic incompleteness in various magnitude bins. Since their incompleteness depends rather little on magnitude, we adopt their cumulative incompleteness measured for $R \leq 20.5$. This completeness has then been corrected in order to take into account the artificial reduction of the number of galaxies due to the presence of a bright star in the cluster field. Also, since we do not wish to consider galaxies inside substructures, we also have to correct the completeness to account for the removal of the substructures. We divided the cluster in radial bins and counted the galaxies inside each bin. In the bins where the presence of the star and the removal of substructures causes a lack of detection, the area of the bin is artificially reduced, and the mean density of galaxies is computed in the remainder of the bin. This value is then assigned to the whole bin.

The RED galaxy number density profile is well fitted by a projected NFW profile (Bartelmann 1996; Łokas \& Mamon 2001). The fit is an MLE fit performed on all RED members that provide a scale radius $0.95 \pm 0.14 \mathrm{Mpc}$. The ALL and BLUE samples are less concentrated, the values of the scale radius being $1.84 \pm 0.25 \mathrm{Mpc}$ for the ALL sample and $16 \pm 11 \mathrm{Mpc}$ for

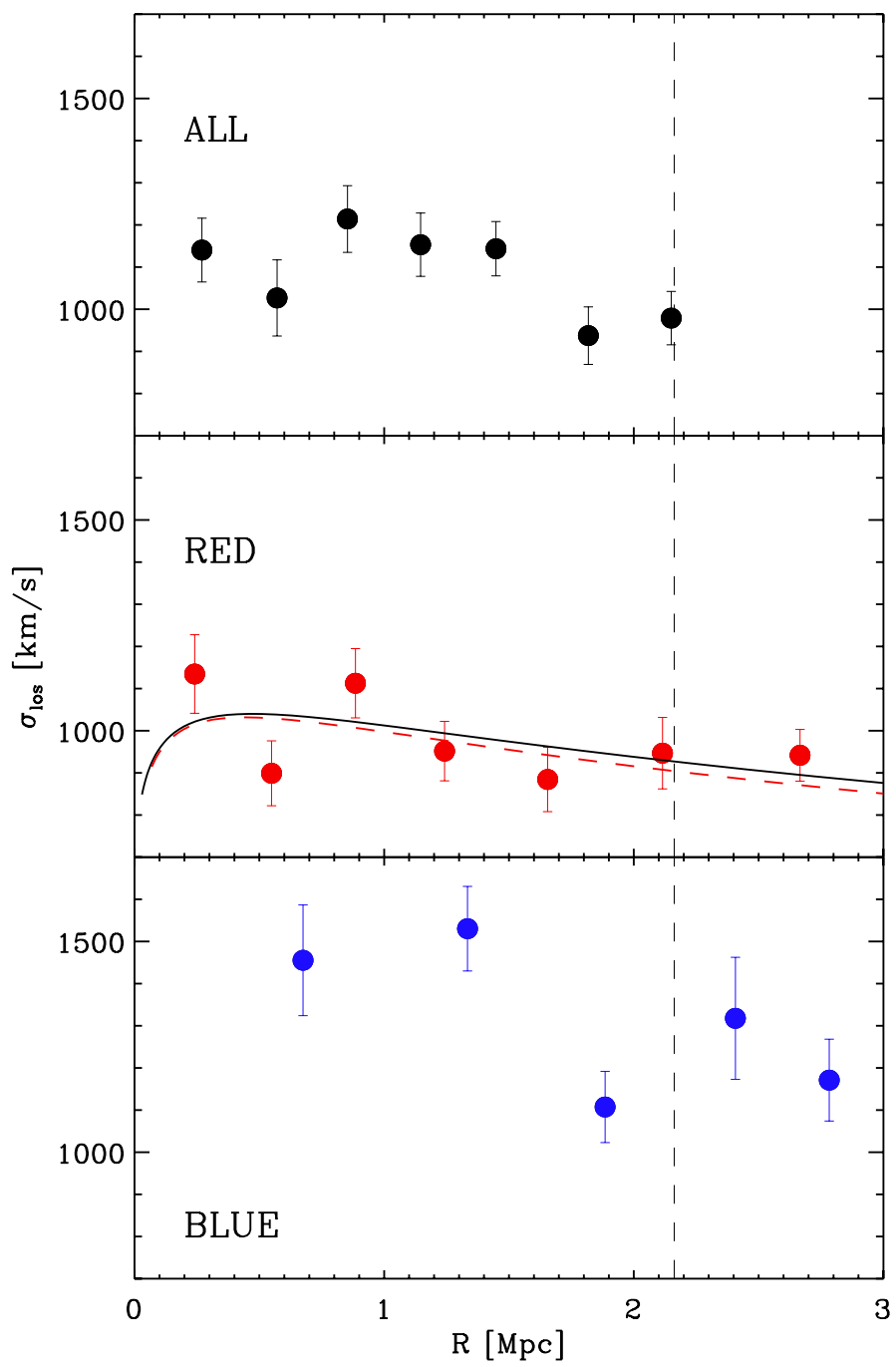

Fig. 5. Velocity dispersion profiles for the ALL, RED, and BLUE samples. For the RED sample we also show the best-fit profile coming from the DK analysis (black), and the profile computed after the MAMPOSSt analysis (dashed red). The dashed vertical line locates the virial radius of the combined model (see Sect. 4).

the BLUE sample. A KS test (e.g. Press et al. 1993) provides an estimate of the reliability of these fits. The probabilities of obtaining greater discrepancy by chance for the RED and BLUE samples are $P=0.95$ and 0.20 , respectively, indicating that the model adequately fits the data. However, for the ALL sample, the corresponding probability is only $P=0.05$, indicating that the model only marginally fits the data. In Fig. 4 the surface number density profiles for the different samples are shown. The scale radius for the BLUE sample is very high and is due to a very flat distribution of these galaxies.

\section{Mass profiles}

\subsection{Mass profiles obtained from the different methods}

We used the velocities of the galaxies within the "first guess" virial radius (see Sect. 2) to compute the mass profile of A2142. In Fig. 5, the velocity dispersion profiles are shown, along with the best-fit profiles coming from the DK and MAMPOSSt analyses.

The DK technique assumes a constant value for the anisotropy, while we have chosen two profiles for the anisotropy 
Table 3. Virial quantities of Abell 2142 obtained from different techniques.

\begin{tabular}{lcllllll}
\hline \hline Method & Sample & $M_{200}\left[10^{15} M_{\odot}\right]$ & $r_{200}[\mathrm{Mpc}]$ & $r_{s}[\mathrm{Mpc}]$ & $c$ & $\sigma_{r} / \sigma_{\mathrm{t}}$ & $\beta$ \\
\hline Caustic $\left(\mathcal{F}_{\beta}=0.5\right)$ & ALL & $1.26_{-0.42}^{+0.54}$ & $2.17_{-0.28}^{+0.27}$ & $0.58_{-0.10}^{+0.12}$ & $3.7 \pm 0.9$ & & \\
DK & RED & $1.32_{-0.21}^{+0.11}$ & $2.20_{-0.12}^{+0.06}$ & $0.93_{-0.10}^{+0.39}$ & $2.4 \pm 0.6$ & $1.0_{-0.04}^{+0.20}$ & $0.0_{-0.1}^{+0.3}$ \\
MAMPOSSt & RED & $1.28_{-0.49}^{+0.14}$ & $2.18_{-0.32}^{+0.08}$ & $0.83_{-0.35}^{+1.73}$ & $2.6_{-1.9}^{+2.0}$ & $1.0_{-0.20}^{+0.50}$ & $0.0 \pm 0.6$ \\
\hline Kinematics & & $1.31_{-0.23}^{+0.26}$ & $2.19 \pm 0.14$ & $0.64 \pm 0.17$ & $3.4 \pm 0.9$ & & \\
\hline X-ray & & $1.11_{-0.31}^{+0.55}$ & $2.08_{-0.22}^{+0.30}$ & $0.74 \pm 0.31$ & $2.8 \pm 1.1$ & & \\
WL & $1.24_{-0.16}^{+0.18}$ & $2.16 \pm 0.10$ & $0.51 \pm 0.08$ & $4.3 \pm 0.7$ & & \\
\hline Combined model & & $1.25 \pm 0.13$ & $2.16 \pm 0.08$ & $0.54 \pm 0.07$ & $4.0 \pm 0.5$ & & \\
\hline
\end{tabular}

Notes. Values of virial mass, virial radius, mass scale radius, concentration, and two measures of the velocity anisotropy, for different techniques. Also shown are the average value of the kinematical techniques after symmetrizing the errors and the value of the combined model, obtained as the result of the average of all the values coming from the different techniques (see Sect. 5 for the average procedure). X-ray values come from Akamatsu et al. (2011), weak lensing (WL) from Umetsu et al. (2009). Both for X-ray and WL we had the values and the errors of the virial radius and the concentration: we have symmetrized these errors and propagated them to obtain the estimates of the errors on the mass scale radii.

model in MAMPOSSt, a constant value and a Tiret profile $\beta(r)=$ $\beta_{0}+\left(\beta_{\infty}-\beta_{0}\right) r /\left(r+r_{\text {anis }}\right)$. Here, we set $\beta_{0}=0$ (inner isotropy) and set $r_{\text {anis }}$ to the scale radius of the galaxy's number density profile. The maximum values of the likelihoods are similar when using the two anisotropy models, therefore for the sake of simplicity we consider only the case of a constant velocity anisotropy. In Sect. 5, we compute the anisotropy profile for the RED sample and find that indeed it is compatible with a constant value.

We also tried to assume different mass profiles and velocity anisotropy models in MAMPOSSt, namely a Burkert (Burkert 1995), a Hernquist (Hernquist 1990) and a softened isothermal sphere profile (e.g. Mamon 1987; Geller et al. 1999), all with both constant and Tiret anisotropy profiles. However our dataset is not large enough to allow us to distinguish between these different models. All provide acceptable fits. As a consequence, the resulting estimates of virial mass and mass profile concentration are very similar to the case of NFW mass profile with constant anisotropy, with differences of very few percent. We therefore only considered the NFW model for the mass profile.

The results are summarized in Table 3. Figure 6 shows the detailed results of our MAMPOSSt MCMC analysis. The mass scale radius is not well constrained by MAMPOSSt. This does not affect the subsequent analysis, since in Sect. 4.2, we perform a weighted mean of the results from the different methods.

In Fig. 7, we show the mass profiles obtained from the different methods, along with the virial values of mass and radius. The results coming from the X-ray (Akamatsu et al. 2011) and weak lensing (WL, Umetsu et al. 2009) analysis are also shown.

\subsection{Combined mass profile}

We combined the constraints from the different mass modelling methods to build a combined mass profile, assuming again an NFW density profile. We attempted to give the same weight to kinematics, X-ray, and WL in the final estimate of the parameters, so we computed single values coming from kinematical techniques for the mass scale radius and virial radius. For this, we took the mean of the values $r_{s}$ and $r_{200}$ of the different methods, inversely weighting by the symmetrized errors. Since the measures of these two quantities by the various methods are not independent (as they are based on essentially the same data sets), we multiplied the error on the average by $\sqrt{3}, 3$ being the number of values used to compute the average. In fact, the usual error on the weighted average decreases like the square root of the number of values.

The mean value and its error are shown in the left panels of Fig. 8. In the right panels of Fig. 8, we plot the values of mass scale and virial radius obtained from the three independent methods: kinematics, X-ray, and WL. The average errorweighted value and its error, this time computed without multiplication factor (since the three measures are independent), are $r_{200}=2.16 \pm 0.08 \mathrm{Mpc}, r_{s}=0.54 \pm 0.07 \mathrm{Mpc}$.

\section{Velocity anisotropy profiles}

Although with DK and MAMPOSSt we have assumed some models for the velocity anisotropy profile, we now wish to determine it in a non-parametric way using the Jeans equation. For this, we use the mass profile we obtained by combining the information coming from the three dynamical methods, X-ray and WL. The Jeans equation contains four unknown quantities, therefore to solve it we need three other relations, namely the Abell integrals to relate the projected number density and velocity dispersion to the real ones and assume a mass profile for the cluster. This anisotropy inversion was first solved by Binney \& Mamon (1982), but several other authors have provided simpler algorithms. We follow the approach of Solanes \& Salvador-Solé (1990), and we tested the results by comparing them with those obtained following the approach of Dejonghe \& Merritt (1992). Once the mass profile is specified, this procedure is fully nonparametric. In fact, instead of fitting the number density profile, we binned and smoothed it with the LOWESS technique (see e.g. Gebhardt et al. 1994). We then obtained the 3D number density profile by using Abel's equation (e.g. Binney \& Mamon 1982). In the same way, we smoothed the binned $\sigma_{\text {los }}$ profile. This procedure requires the solution of integrals up to infinity. Mamon et al. (2010) show that a $3 \sigma$ clipping removes all the interlopers beyond 19 virial radii. Therefore, an extrapolation up to such a distance is enough to solve the integrals having infinity as the integration limit. We used $30 \mathrm{Mpc}$ as the maximum radius of integration, and extrapolate the smoothed profiles up to this limit. A factor-2 change in the upper limit of integration does not affect our results in a significant way.

The result of the anisotropy inversion is shown in Fig. 9. The confidence levels were obtained by estimating two error contributions. One contribution comes from the uncertainties in the surface density and $\sigma_{\text {los }}$ profiles. Since $80 \%$ of the relative 

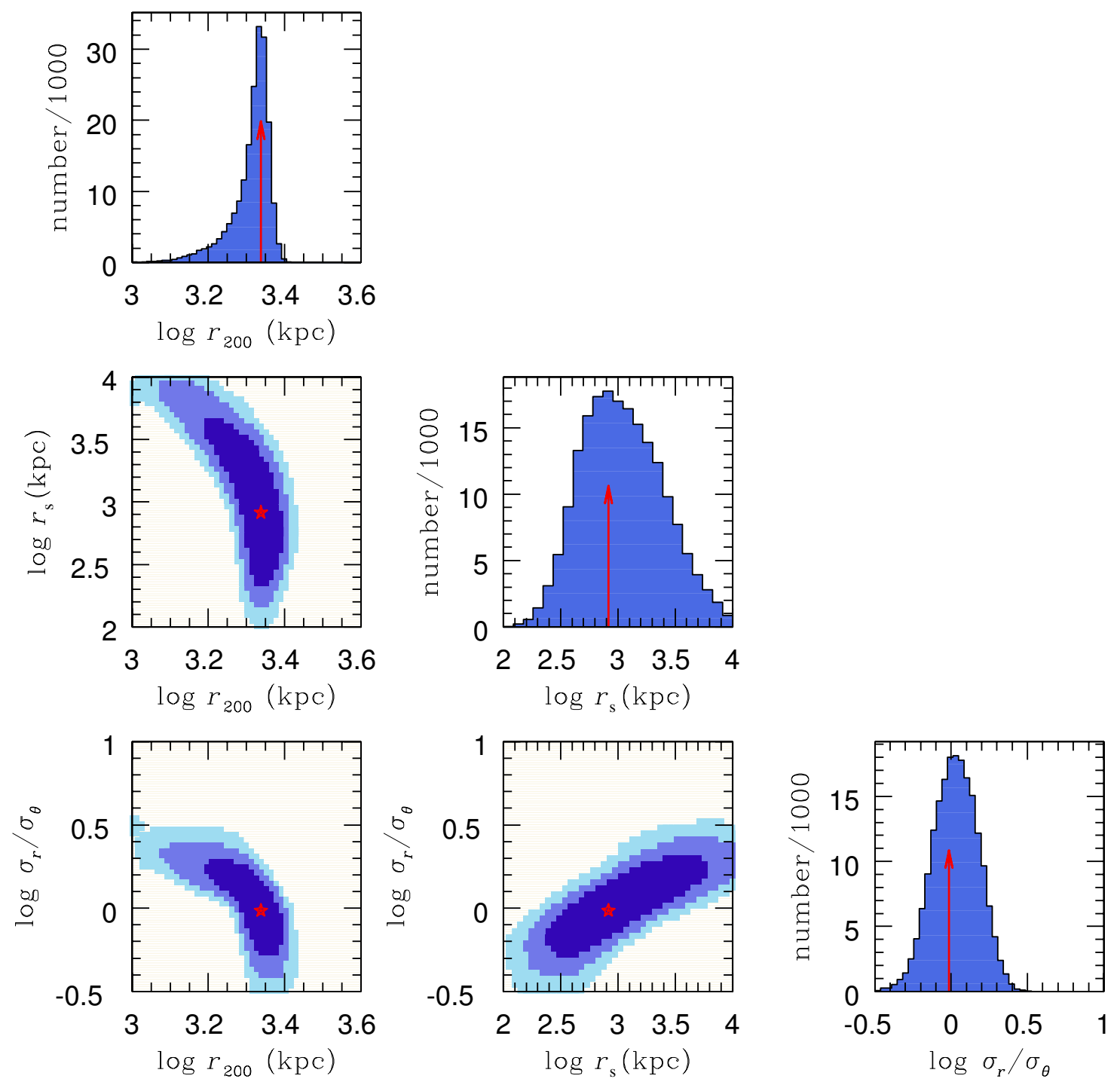

Fig. 6. Parameter space and probability distribution functions for the virial radius, mass profile scale radius, and velocity anisotropy, as found by MAMPOSSt. The coloured regions are the 1,2,3 $\sigma$ confidence regions, while the red stars and the red arrows locate the best-fit values. These are based upon an MCMC analysis with 6 chains of 40000 elements each, with the first 5000 elements of each chain removed (this is the burn-in phase that is sensitive to the starting point of the chain). The priors were flat within the range of each panel, and zero elsewhere.

uncertainty of the product $\Sigma \sigma_{\text {los }}^{2}$ comes from the uncertainty of $\sigma_{\text {los }}$ (Trilling et al., in prep.), we only considered the error contribution from the latter. It is virtually impossible to propagate the errors on the observed $\sigma_{\text {los }}$ through the Jeans inversion equations to infer the uncertainties on the $\beta$ profile solution. We then proceeded to estimate these uncertainties the other way around. We modify the $\beta$ profile in two different ways: 1) $\beta(r) \rightarrow \beta(r)+S+T r$, and 2) $\beta(r) \rightarrow J \beta(r)+Y$, using a wide grid of values for the constants, respectively $(S, T)$ and $(J, Y)$. Using the mass and anisotropy profiles, it is then possible to determine $\sigma_{r}(r)$ and then the $\sigma_{\text {los }}$ profile (e.g. Mamon \& Łokas $2005 b)$. The range of acceptable $\beta$ profiles is determined by a $\chi^{2}$ comparison of the resulting $\sigma_{\text {los }}$ profiles with the observed one.

In addition, another source of uncertainty on the $\beta$ profile solution comes from the uncertainty in the mass profile. This is estimated by running the anisotropy inversion for four different mass profiles corresponding to the combination of allowed values of virial and mass scale radii within $1 \sigma$. The profiles obtained modifying the mass profile (not shown) lie within the confidence interval of the main result, so that the confidence interval represents the uncertainty on the anisotropy profile well.
The ALL sample $\beta(r)$ depends weakly on radius: the innermost region is compatible with isotropy, while the anisotropy is increasingly radial at large radii. The RED sample is compatible with isotropy at all radii. The difference between the two samples is almost entirely due to the BLUE galaxies, the anisotropy of which is compatible with isotropy in the centre, then becomes rapidly radially anisotropic, and finally flattens at radii $>1 \mathrm{Mpc}$.

As a check, we compare the values of $\beta$ obtained from the anisotropy inversion with the best-fit results of DK and MAMPOSSt. In these techniques, we assumed a constant value of the anisotropy for the RED sample, which appears to be a good assumption given the results of $\beta$ after the inversion. The value estimated by both DK and MAMPOSSt is $\beta=0.0$, consistent within the uncertainties with the $\beta$ profile shown in Fig. 9.

\section{6. $Q(r)$ and $\beta-\gamma$ relations}

Since our anisotropy inversion provides us with the radial variations of $\sigma(r)=\sqrt{\sigma_{r}^{2}+2 \sigma_{\mathrm{t}}^{2}}, \beta(r)$ (from which $\sigma_{r}(r)$ follows), we can take advantage of the results just found for the galaxy 


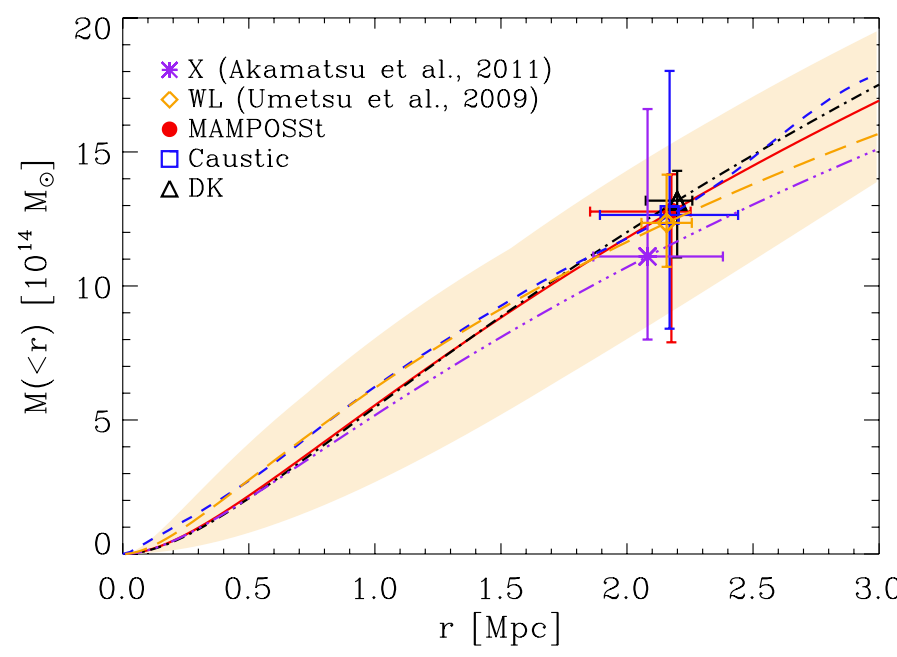

Fig. 7. Mass profiles computed from the different methods. The black dash-dotted line and the triangle with error bars refer to DK technique, the dashed blue line and blue square to the caustic method, the solid red line and red point to MAMPOSSt. The symbols with error bars refer to the virial mass and radius. The purple asterisk with error bars and the purple dash triple dotted line are the result of the X-ray analysis, while the orange diamond with a long dashed line is the one coming from weak lensing analysis. The shaded area is the $1 \sigma$ confidence region of the mass profile according to the MAMPOSSt results.

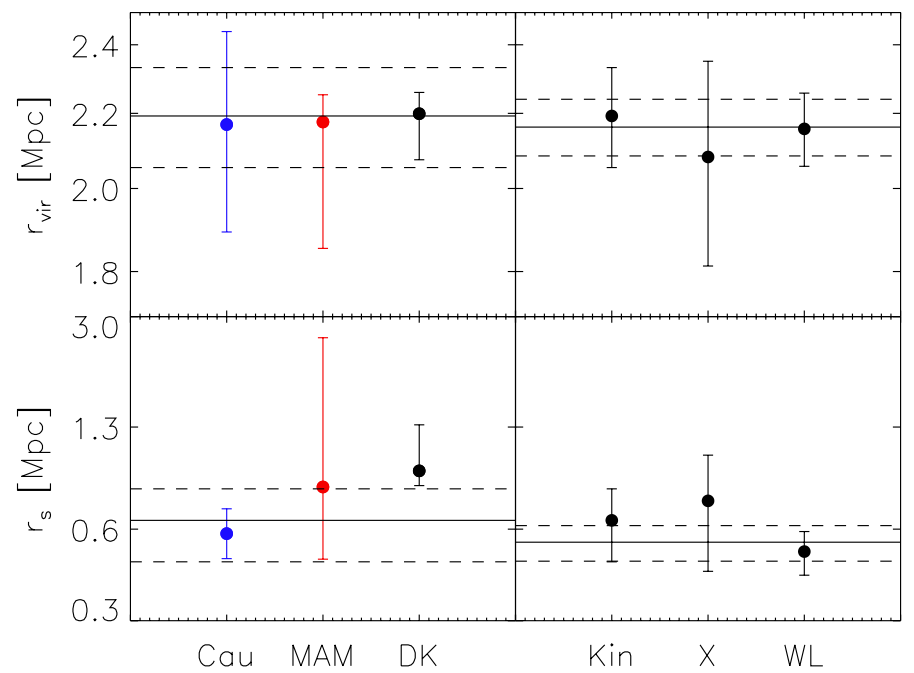

Fig. 8. Virial (top panels) and mass scale (bottom panels) radius for all the methods. Left panels: blue diamonds are values obtained from the caustic technique, red ones for MAMPOSSt, and black ones for DK (from left to right, respectively). The average value and its error are the solid and dashed lines, respectively. See the text for the computation of the error. Right panels: values obtained from the kinematical analysis, $\mathrm{X}$-ray and WL (from left to right, respectively). The average value and its error are the solid and dashed lines, respectively.

populations of A2142 to test the PPSD profile and the relation linking the logarithmic slope of the density profile and the anisotropy $\beta(r)$.

Both the PPSD and $\beta-\gamma$ relations were derived from dissipationless single-component simulations. It is therefore not clear whether the power-law PPSD and the linear beta-gamma relation, both found for the particles of single component dark matter (DM)-only simulations, will be obtained when using galaxies to measure the velocity dispersion or velocity anisotropy and

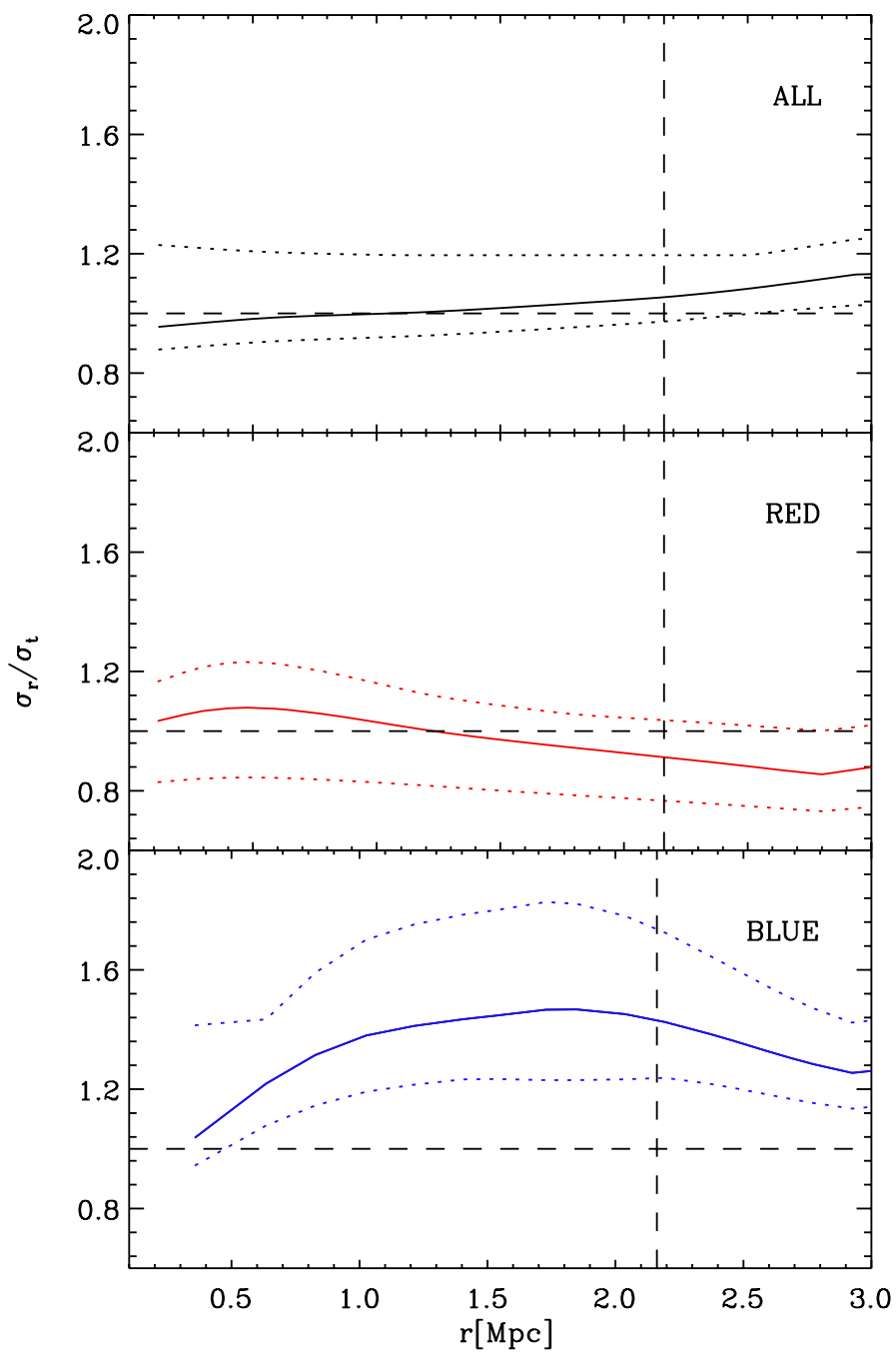

Fig. 9. Velocity anisotropy profiles for the ALL, RED, and BLUE samples. The solid line is the result of the inversion of the Jeans equation, while the dotted lines are the $1 \sigma$ confidence intervals. The vertical dashed line locates the virial radius.

whether one should use the total density or the galaxy number density in these two relations. We discuss this further in Sect. 7.

\subsection{Use of the total matter density profile}

We begin by adopting the total density profile $\rho(r)$. We compute both the PPSD profile $Q(r)=\rho / \sigma^{3}$ and its radial counterpart $Q_{r}(r)=\rho / \sigma_{r}^{3}$. In Fig. 10, we show, for the different tracers (ALL, RED, BLUE), the radial profile of $Q(r)$ (left panels) and $Q_{r}(r)$ (right panels) within the virial radius. To compute the errors on the best-fit slope parameters, we have assumed that the number of independent $Q$ and $Q_{r}$ values are the same as those of the observed velocity dispersion profile (see Fig. 5).

Assuming a power-law behaviour of the PPSD profile, as suggested by Dehnen \& McLaughlin (2005), we fit the profiles of both $Q(r)$ and $Q_{r}(r)$ in two ways, either keeping the exponent fixed to the values found for haloes in $\triangle \mathrm{CDM}$ simulations by Dehnen \& McLaughlin (2005) or considering it as a free parameter. In both cases the normalization is left as a free parameter. In Table 4 the results of such fits are shown. The $Q(r)$ profile for the RED sample is consistent within less than $2 \sigma$ with the $r^{-1.84}$ relation by Dehnen \& McLaughlin (2005). The fit of the profile with a linear relation in the log-log plane is compatible 
Table 4. Best-fit parameters of the PPSD profile.

\begin{tabular}{|c|c|c|c|c|}
\hline & \multicolumn{2}{|l|}{$Q(r)$} & \multicolumn{2}{|l|}{$Q_{r}(r)$} \\
\hline & $\begin{array}{c}A \\
{\left[M_{\odot} \mathrm{Mpc}^{-3} \mathrm{~km}^{-3} \mathrm{~s}^{3}\right]}\end{array}$ & $B$ & $\begin{array}{c}A \\
{\left[M_{\odot} \mathrm{Mpc}^{-3} \mathrm{~km}^{-3} \mathrm{~s}^{3}\right]} \\
\end{array}$ & $B$ \\
\hline \multicolumn{5}{|c|}{ Fixed slope } \\
\hline $\begin{array}{l}\text { ALL } \\
\text { RED } \\
\text { BLUE } \\
\end{array}$ & $\begin{array}{l}5534 \pm 314 \\
7727 \pm 391 \\
1753 \pm 294 \\
\end{array}$ & $\begin{array}{l}-1.84 \\
-1.84 \\
-1.84 \\
\end{array}$ & $\begin{array}{c}25071 \pm 3341 \\
38484 \pm 5622 \\
3998 \pm 1084 \\
\end{array}$ & $\begin{array}{l}-1.92 \\
-1.92 \\
-1.92 \\
\end{array}$ \\
\hline \multicolumn{5}{|c|}{ Free slope } \\
\hline $\begin{array}{l}\text { ALL } \\
\text { RED } \\
\text { BLUE } \\
\end{array}$ & $\begin{array}{l}6342 \pm 367 \\
8034 \pm 411 \\
3121 \pm 793 \\
\end{array}$ & $\begin{array}{l}-2.28 \pm 0.11 \\
-2.00 \pm 0.09 \\
-2.97 \pm 0.50 \\
\end{array}$ & $\begin{array}{c}29175 \pm 4223 \\
38881 \pm 5665 \\
5413 \pm 1810 \\
\end{array}$ & $\begin{array}{l}-2.27 \pm 0.24 \\
-1.77 \pm 0.23 \\
-2.60 \pm 0.67\end{array}$ \\
\hline & \multicolumn{2}{|c|}{$Q(r) G A L$} & \multicolumn{2}{|c|}{$Q_{r}(r) G A L$} \\
\hline & $\begin{array}{c}A \\
{\left[10^{-9} \mathrm{Mpc}^{-3} \mathrm{~km}^{-3} \mathrm{~s}^{3}\right]}\end{array}$ & $B$ & $\begin{array}{c}A \\
{\left[10^{-9} \mathrm{Mpc}^{-3} \mathrm{~km}^{-3} \mathrm{~s}^{3}\right]}\end{array}$ & $B$ \\
\hline \multicolumn{5}{|c|}{ Fixed slope } \\
\hline $\begin{array}{l}\text { ALL } \\
\text { RED } \\
\text { BLUE } \\
\end{array}$ & $\begin{array}{l}6.82 \pm 0.68 \\
3.62 \pm 0.46 \\
0.98 \pm 0.23 \\
\end{array}$ & $\begin{array}{l}-1.84 \\
-1.84 \\
-1.84 \\
\end{array}$ & $\begin{array}{c}28.49 \pm 5.37 \\
13.23 \pm 3.21 \\
1.30 \pm 0.47 \\
\end{array}$ & $\begin{array}{l}-1.92 \\
-1.92 \\
-1.92 \\
\end{array}$ \\
\hline \multicolumn{5}{|c|}{ Free slope } \\
\hline $\begin{array}{l}\text { ALL } \\
\text { RED } \\
\text { BLUE } \\
\end{array}$ & $\begin{array}{c}10.19 \pm 25.60 \\
8.21 \pm 17.01 \\
0.88 \pm 1.81 \\
\end{array}$ & $\begin{array}{l}-1.09 \pm 0.15 \\
-0.90 \pm 0.14 \\
-0.90 \pm 0.61\end{array}$ & $\begin{array}{c}46.94 \pm 7.34 \\
40.48 \pm 6.69 \\
1.52 \pm 0.55\end{array}$ & $\begin{array}{l}-1.09 \pm 0.26 \\
-0.71 \pm 0.25 \\
-0.52 \pm 0.74\end{array}$ \\
\hline
\end{tabular}

Notes. The PPSD profile is parametrized as $Q(r)=A \cdot r^{B}$. The first panel at the top shows the results of the fit of $Q(r)$ and $Q_{r}(r)$ for the different samples, both when keeping fixed the exponent to the values suggested by Dehnen \& McLaughlin (2005), and when considering the exponent as a free parameter. In the bottom panel (which is the one identified by $Q(r) G A L$ and $Q_{r}(r) G A L$ ) the same quantities are shown, but they refer to the PPSD computed using the galaxy number density profile instead of the total matter density profile.

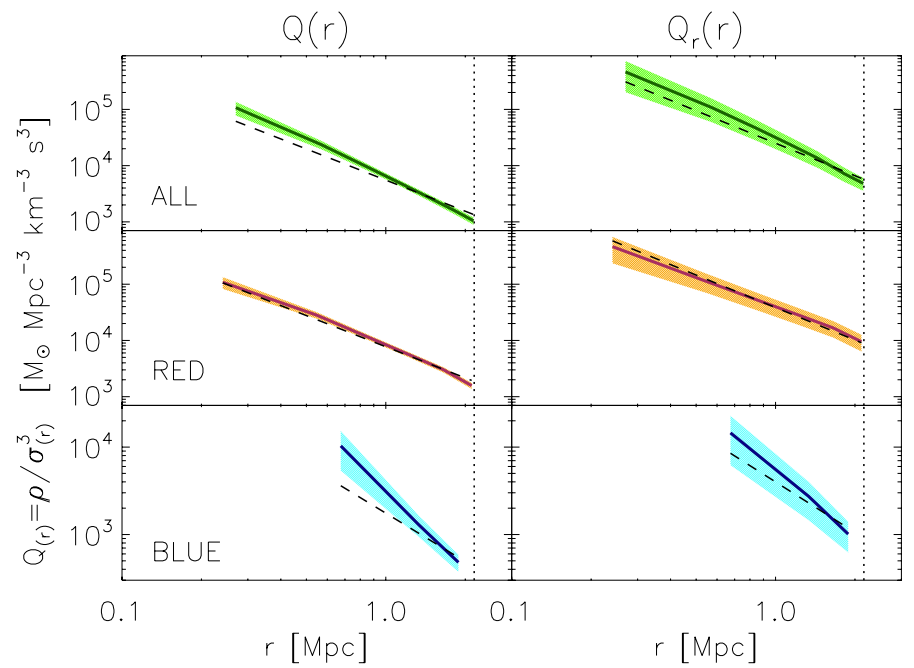

Fig. 10. Radial profiles of $Q$ (left columns) and $Q_{r}$ (right columns) within the virial radius, and the $1 \sigma$ confidence regions (shaded areas), for different types of member tracers: green for the ALL sample (top panels), red for the RED sample (middle panels), and blue for the BLUE sample. The shaded areas represent the propagation of the errors associated with $\rho, \sigma$ and $\sigma_{r}$. The dashed lines are the power-law relations $Q(r) \propto r^{-1.84}$ and $Q_{r}(r) \propto r^{-1.92}$ found by Dehnen \& McLaughlin (2005) on numerically simulated haloes. The vertical dotted lines locate the virial radius of the combined model (see Sect. 4).

with the theoretical value -1.84 within $1.7 \sigma$. On the other hand, for the BLUE sample, the slope of the PPSD is steeper than the theoretical expectation.
The $\sigma_{r}$ profile is affected by larger uncertainties than the $\sigma$ profile, because the former combines the uncertainties from the latter and $\beta(r)$, which are the parameters produced by the anisotropy inversion algorithm of Solanes \& Salvador-Solé (1990). It is therefore not surprising that, within the uncertainties, the $Q_{r}$ profiles of all three samples appear consistent with the theoretical expectation for simulated $\Lambda \mathrm{CDM}$ haloes (Dehnen $\&$ McLaughlin 2005), $Q_{r} \propto r^{-1.92}$. We note, however, that the agreement is quite remarkable (within $0.3 \sigma$ ) for the RED sample.

Ludlow et al. (2010) warn against fitting the pseudo phasespace density profile outside the scale radius, because of the upturn they find in the $Q(r)$ profile in the outer regions. However, for our three samples, none of the $Q(r)$ and $Q_{r}(r)$ profiles show significant curvature in log-log space.

In Fig. 11, we show the anisotropy-density slope relation. The $\beta-\gamma$ relation of the ALL sample matches the one found by Hansen \& Moore (2006) closely in single-component dissipationless simulations (cosmological and academic); however, the $\beta-\gamma$ relation for the RED sample shows curvature, with lower values of $\beta$ at the steeper slopes (larger radii) than found in simulations by Hansen \& Moore (2006).

It can be proven that all multicomponent spherical systems with positive phase-space distribution function, for which 1) the density of a component is a separable function of total gravitational potential and radius; 2) $\beta(0) \leq 1 / 2$ (i.e. are not too much radially anisotropic at the center), necessarily satisfy $\beta(r)<-\gamma(r) / 2$, where the velocity anisotropy $\beta$ and the logarithmic slope of density $\gamma$ are for that component, as shown in Ciotti \& Morganti (2010; see also Van Hese et al. 2011). It is not clear 


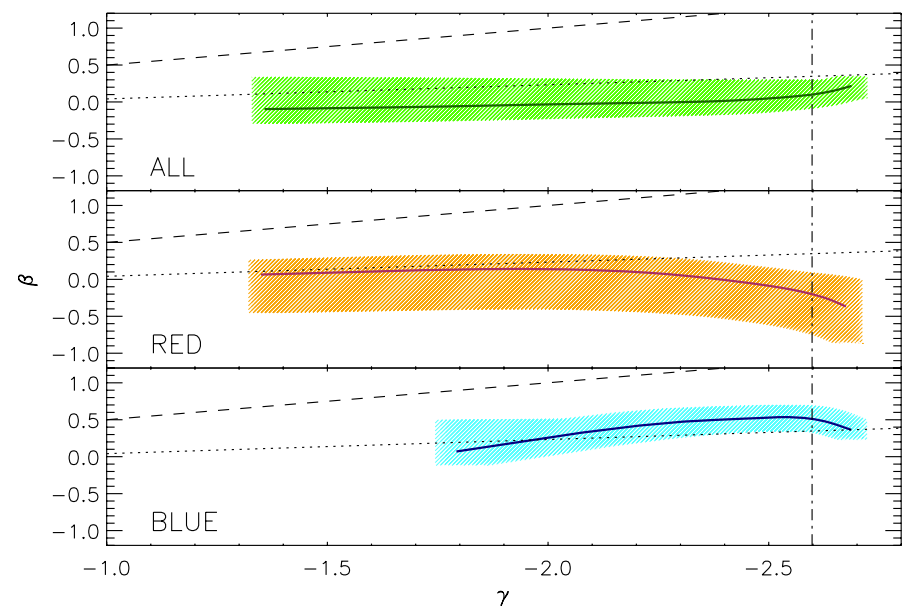

Fig. 11. Velocity anisotropy versus logarithmic slope of the total density profile. The samples are ALL galaxies (top), RED (middle), and BLUE galaxies (bottom panel). The shaded areas are the $1 \sigma$ confidence regions. The $\beta-\gamma$ relation found by Hansen \& Moore (2006) for singlecomponent dissipationless simulations is shown as the dotted lines. The dashed line is the limit below which the relation by Ciotti \& Morganti (2010) holds. The vertical dot-dashed line locates the value of $\gamma$ at the virial radius.

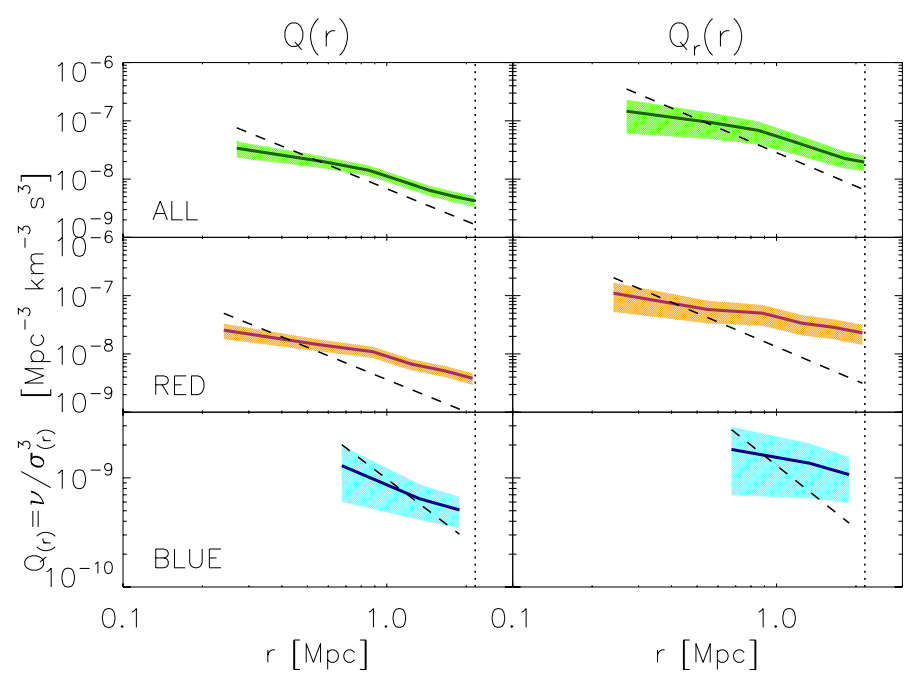

Fig. 12. Same as Fig. 10, but now using the radial profiles of galaxy number density instead of total mass density to estimate the PPSD.

whether the galaxy components of clusters of galaxies have such separable densities.

\subsection{Use of the tracer density profile}

As we discuss at length in Sect. 7, it is not obvious that one should use the total mass density profile rather than the tracer number density profile in evaluating the PPSD and the $\beta-\gamma$ relations, when we want to compare them to those found in numerical simulations. As a result, we now repeat repeat our analyses of the PPSD and the $\beta-\gamma$ relations, replacing the total mass density with the number density of the tracer of the sample.

In Fig. 12, we show the PPSD computed using the galaxy number density profile instead of the total matter density one. For all three samples, both $Q(r)$ and $Q_{r}(r)$ remain as power laws, but are considerably shallower than the relation found by Dehnen \& McLaughlin (2005) on simulated ACDM haloes.

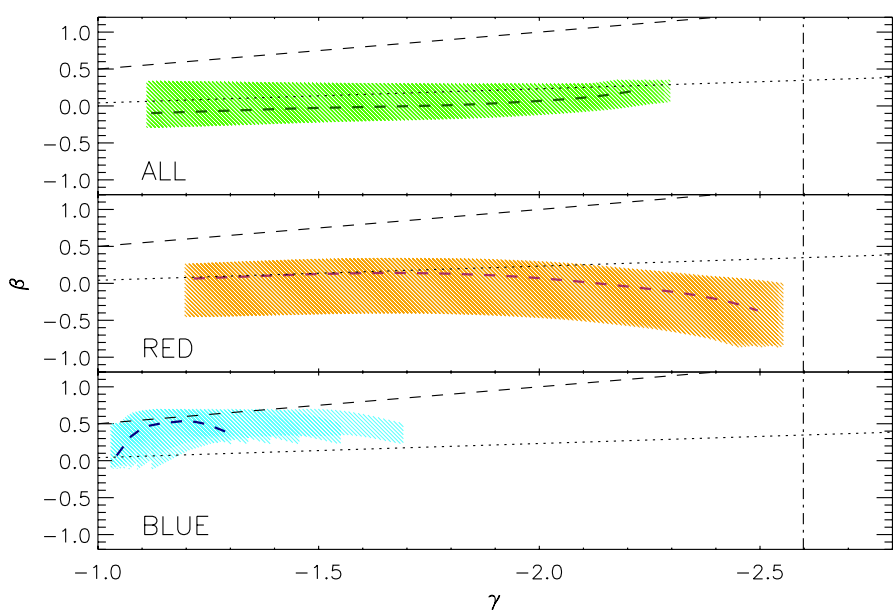

Fig. 13. Same as Fig. 11, but now using the radial profiles of galaxy number density of the three samples instead of total mass density to estimate the slope.

In Fig. 13, we show the $\beta-\gamma$ relation computed using the galaxy number density profile instead of the total matter density one. The behaviour does not change significantly from the case of the $\beta-\gamma$ relation computed using the total matter density profile: the overall shapes of the profiles are similar, but the BLUE sample now presents a noisier profile, while ALL and RED profiles are shifted towards higher values of $\gamma$, reflecting the shallower trend of the galaxy number density profile with respect to the matter density one. We discuss these results below.

\section{Discussion}

\subsection{Dynamical status}

Munari et al. (2013) report the scaling relation between the virial mass of clusters and the velocity dispersion of the member galaxies within the virial sphere. Using the most realistic ("AGN") hydrodynamical simulation at their disposal, they find $\sigma_{1 \mathrm{D}}=1177\left[h(z) M_{200} / 10^{15} M_{\odot}\right]^{0.364}$ for the galaxies within the virial sphere, where $\sigma_{1 \mathrm{D}}$ is the total $3 \mathrm{D}$ velocity dispersion within $r_{200}$, divided by $\sqrt{3}$. The analysis was carried out in the $6 \mathrm{D}$ phase space, so is immune to projection effects. The statistical nature of their relation suggests that it should hold for real, observed, and relaxed systems. As a test, we checked the consistency of the velocity dispersion - mass relation found by Munari et al. (2013) with our findings for A2142. The values of virial mass obtained with this relation are: $1.42 \times 10^{15} M_{\odot}$ for the ALL sample, $1.07 \times 10^{15} M_{\odot}$ for the RED sample, and $2.50 \times 10^{15} M_{\odot}$ for the BLUE sample. The values obtained for the ALL and RED samples agree, within the uncertainties, with the combined value of the mass of A2142. This seems to indicate that RED cluster members are in, or very close to, equilibrium. The large difference obtained for the BLUE cluster members warns against using the blue galaxy LOS velocity dispersion as a proxy for the cluster mass.

A glance at Table 3 indicates that our different estimates of the mass concentrations are bimodal: the caustic and weak lensing have values $\simeq 4$, while those for the DK, MAMPOSSt, and X-ray methods are $<3$. Could these lower mass concentrations found by methods based upon internal kinematics be a sign that A2142 is out of dynamical equilibrium? The substructures found by Owers et al. (2011) and the results by Rossetti et al. (2013) on the importance of the mergers undergone by A2142 


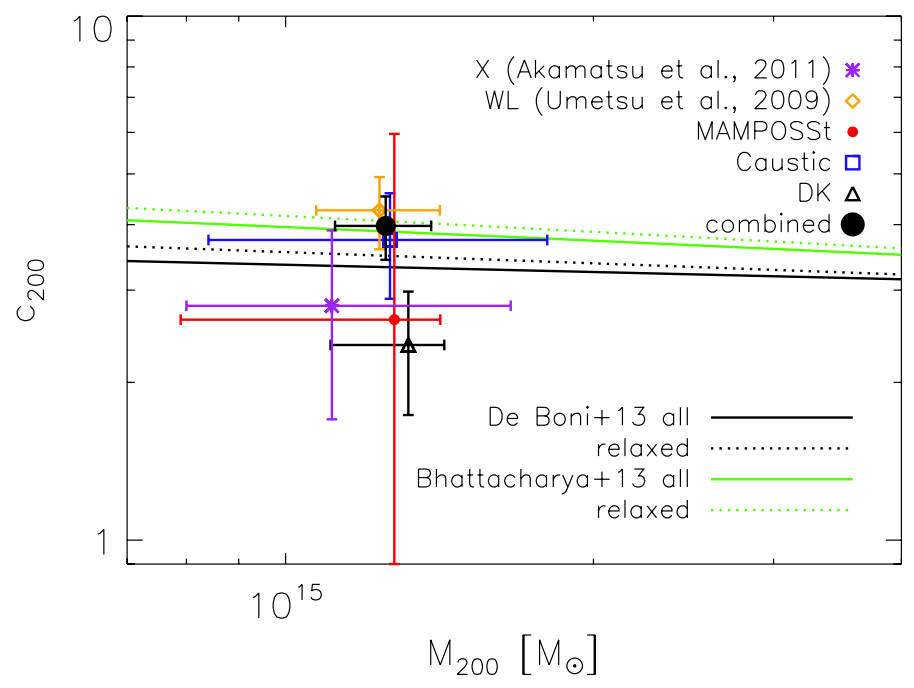

Fig. 14. Concentration-mass relation, with respect to an overdensity 200 times the critical one. Purple asterisk refers to the X-ray values by Akamatsu et al. (2011), orange diamond to the WL values by Umetsu et al. (2009), small red circle refers to the values obtained by MAMPOSSt, blue square by the caustic method, black triangle by DK, the big black circle to the values of the combined model. Lines are the theoretical predictions, and in black the relations by De Boni et al. (2013) when considering all (solid) and relaxed (dotted) clusters. In green the relations by Bhattacharya et al. (2013) when considering all (solid) and relaxed (dotted) clusters.

suggest that full relaxation is to be excluded. On the other hand, the agreement on the virial radius amongst the different methods and with the results from X-ray and lensing (the latter does not require equilibrium) suggests that $\mathrm{A} 2142$ is not far from dynamical equilibrium.

In Fig. 14, the concentration - mass relation for A2142 is shown along with theoretical relations by Bhattacharya et al. (2013) and De Boni et al. (2013) based on cosmological $N$-body and hydrodynamical simulations, respectively. The value of the combined model $\left[M_{200}=(1.25 \pm 0.13) \times 10^{15} M_{\odot}\right.$ and $c=$ $4.0 \pm 0.5$ ] agrees within $1 \sigma$ with the "relaxed" case of De Boni et al. (2013), while it is in excellent agreement with both the relations by Bhattacharya et al. (2013). This strengthens the scenario of A2142 being very close to equilibrium.

\subsection{Mass density profile}

Previous studies based on the kinematics of galaxies in clusters have shown that galaxy populations have similar concentrations to those of the total matter, or slightly smaller, blue galaxies being instead much less concentrated (see, e.g. Biviano \& Girardi 2003; Katgert et al. 2004). On the other hand, Biviano \& Poggianti (2009) found in the ENACS clusters that the red galaxy population has a concentration that is as much as 1.7 times lower for the total matter density profile. Here, we find that the scale radius for the RED galaxy number density profile $(0.95 \mathrm{Mpc})$ is 1.8 times greater than for the total mass density profile from our combined model, which agrees with the ENACS result. Collister \& Lahav (2005) found, on a stacked sample from the $2 \mathrm{dFGRS}$, values of galaxy concentration comparable to ours, when considering objects as massive as A2142 (see their Fig. 7), although with uncertainties that are too large to distinguish between red and blue samples.

The scale radius of the BLUE population in Abell 2142 appears unusually high, leading to concentrations (using our combined virial radius) of 0.16 (best) or $0.39(+1 \sigma)$, which are much lower than expected from previous studies. Blue galaxies within the virial cones of clusters are more prone to projection effects than red galaxies: Mahajan et al. (2011) analyzed clusters and their member galaxies in the SDSS, using LOS velocities and cosmological simulations to quantify the projection effects. They conclude that $44 \pm 2 \%$ of galaxies with recent (or ongoing) starbursts that are within the virial cone are outside the virial sphere. Since galaxies with recent star formation have blue colours, our BLUE sample includes this recent-starburst subsample, plus perhaps some more galaxies with more moderate recent star formation. Moreover, an analysis of cosmological simulations by Mamon et al. (2010) indicates that there is a high cosmic variance in the fraction of interlopers within the DM particles inside the virial cone. This suggests that the unusually low concentration of the blue galaxy sample could be a sign of an unusually high level of velocity interlopers with low rest-frame velocities in front of and behind Abell 2142.

Wojtak \& Łokas (2010) find a virial radius that corresponds to $r_{200}=2.15_{-0.12}^{+0.10} \mathrm{Mpc}$, in excellent agreement with our different estimates of the virial radius (Table 3 ). On the other hand, they find a mass scale radius $r_{s}=1.0_{-0.2}^{+0.3} \mathrm{Mpc}$ not compatible with our value of the combined model, although in agreement with the results of the DK, MAMPOSSt, and X-ray analyses. Wojtak $\&$ Łokas assumed that the DM and galaxy scale radii were equal. Such an unverified assumption may have biased high their scale radius for the mass distribution. On the other hand, the values of the mass scale radii that we found from DK and MAMPOSSt (0.93 and $0.83 \mathrm{Mpc}$, respectively, see Table 3) are consistent with that of the RED galaxy population used as the tracer $(0.95 \mathrm{Mpc})$, as is in Wojtak \& Łokas, both within the uncertainties.

\subsection{Velocity anisotropy profile}

The velocity anisotropy profile for the ALL sample in the centre is compatible with the one found by Wojtak \& Łokas (2010). In the outer part, at $\simeq 3 \mathrm{Mpc}$, the value of $\sigma_{r} / \sigma_{\theta}$ found by Wojtak \& Łokas (2010) is higher and offset from ours by $1.4 \sigma$. An analysis of a stacked sample of 107 nearby ENACS clusters (Biviano \& Katgert 2004; Katgert et al. 2004) shows that the orbits of ellipticals and S0s (hence red) galaxies are compatible with isotropy, while those of early and late-type spirals have radial anisotropy. At slightly higher redshifts, van der Marel et al. (2000) also find red galaxies close to isotropy. The velocity anisotropy profile for our BLUE sample presents behaviour that lies in between the profiles found in Biviano \& Katgert for the early spirals and the late spirals together with emission line galaxies, suggesting agreement between their findings and ours. The anisotropy profile we found for the ALL sample appears to be consistent with those measured by Lemze et al. (2012) and Mamon et al. (2013) in simulated $\Lambda$ CDM haloes. In simulations, data are usually stacked or averaged, and the scatter in the anisotropy profiles is considerable (see e.g. Lemze et al. 2012; Mamon et al. 2013) and this reflects the variety of configurations of galaxy clusters. A2142 does not present strong deviations from the general trend, because its anisotropy profile is compatible with this scatter.

\subsection{PPSD profile and $\beta-\gamma$ relation}

Biviano et al. (2013) analyzed the pseudo phase-space density on MACS1206, a cluster at $z=0.44$. They find a $Q(r)$ profile with a slope for the blue galaxies in agreement with the predictions of Dehnen \& McLaughlin (2005), at odds with our findings. We 
speculate that this different behaviour might provide a hint of the dynamical history of clusters. In fact, a cluster that has only recently undergone the phase of violent relaxation might present a population of blue galaxies in rough dynamical equilibrium. On the other hand, a cluster that has undergone the violent relaxation phase a long time ago, should have had time to transform its blue galaxies into red ones. Therefore the blue galaxy population would be mainly composed of only recently accreted galaxies, hence not in dynamical equilibrium.

The results found in Sect. 6 raise the question of what is more relevant for galaxy clusters when considering the $\beta-\gamma$ and PPSD relations, the total mass density, or the tracer number density. It is important to recall that these relations were derived for dissipationless, single-component systems. Although dominated by DM, clusters of galaxies contain a non-negligible component made up of dissipative diffuse hot gas. One can argue that the PPSD and $\beta-\gamma$ relations and even the NFW density model found in $\Lambda C D M$ haloes concern the total density profiles, as they would be related to the global gravitational potential (hence total mass profile), violent relaxation, or more generally the mass assembly of clusters through a combination of several major mergers and numerous minor mergers. Alternatively, one can argue that these quantities concern the DM component only and that for the PPSD and $\beta-\gamma$ relations, it is inconsistent to associate the total density profile to the tracer velocity dispersion profile and that one should instead associate the tracer density profile to the tracer velocity dispersion profile.

For giant elliptical galaxies, we now know that the NFW model must concern the DM component, while the $\beta-\gamma$ relation found in single-component dissipationless simulations concerns the observed tracer. Indeed, Mamon \& Łokas (2005a) have shown that the observed inner aperture velocity dispersions are too high to be matched by a single NFW component (while the addition of a stellar Sérsic component matches the observations). Moreover, in the elliptical galaxy remnants of binary mergers of spiral galaxies made of stars, gas, and DM, the $\beta-\gamma$ relation is obeyed well by the stellar component, but not for the DM component (Mamon et al. 2006). Also, the stellar or DM $\beta$ do not relate to the slope of the total mass density profile (Mamon, unpublished).

Extrapolating from our understanding of elliptical galaxies, we find it surprising that the PPSDs that we measure for Abell 2142 match the relations found in $\Lambda$ CDM haloes better when the total density profile is used instead of the density profile of the tracer used to estimate the velocity dispersion. Perhaps one should not expect clusters to behave like elliptical galaxies. Indeed, in comparison with the progenitors of elliptical galaxies, the progenitors of clusters (galaxy groups) have deeper gravitational potentials that prevent cooling and dissipative contraction of gas more effectively. Moreover, cluster-mass haloes grow relatively faster at $z=0$ than galaxy-mass haloes (e.g. van den Bosch 2002), hence are built by more recent mergers than elliptical galaxies, and these mergers, some major, will mix the inner regions. For this reason, the baryonic and DM mass distributions in clusters should be closer than in elliptical galaxies.

While galaxies are biased tracers of the DM velocity dispersion (Munari et al. 2013), if the velocity dispersion profile of the galaxy component is proportional to that of the DM component at all radii (i.e. no velocity bias relative to the DM), then the PPSDs built from the galaxies should have the same slope as the one built from the DM. On the other hand, if the velocity bias of the galaxy component is a function of radius, as found by Wu et al. (2013) in cosmological hydrodynamical simulations of clusters, then the PPSD built from the galaxies will be different from the one built with the DM component (after proper normalization). Since the DM component dominates the gravitational potential of clusters, we infer that the consistency of the PPSD, built with the total density and the velocity dispersion of the RED galaxy component, suggests that the velocity bias of the component of red galaxies outside of substructures is roughly independent of radius.

At all radii, the RED galaxy sample shows somewhat lower $\beta$ for given $\gamma$ (measured with total mass density) than found in simulated haloes. However, the $\beta-\gamma$ relations have been derived using DM-only simulations, which do not take the effects of the presence of baryons into account. Now, if the tangential and radial components of the velocity dispersion of the galaxy population are proportional to those of the DM, then the velocity anisotropy of the galaxy population, written as $\mathcal{A}=\sigma_{r} / \sigma_{\theta}$ should be proportional to that of the DM, but the non-linear function of $\mathcal{A}, \beta=1-1 / \mathcal{A}^{2}$, measured for the galaxies, will not necessarily be proportional to the analogous $\beta$ for the DM. Therefore, any radial variation of $\mathcal{A}$, hence $\beta$, will lead to a bias in the $\beta-\gamma$ relation. Finally, the $\beta-\gamma$ relation may vary from cluster to cluster (Ludlow et al. 2011).

\section{Conclusions}

We have computed the mass and velocity anisotropy profiles of A2142, a nearby $(z=0.09)$ cluster, using the kinematics of cluster galaxies. After a membership algorithm was applied, we considered the sample made of all members (ALL sample), as well as two subsamples, consisting of blue member galaxies (BLUE sample) and red member galaxies that do not belong to substructures (RED sample).

We made use of three methods based on the kinematics of galaxies in spherical clusters: DK, MAMPOSSt and Caustic (see Sect. 3). The mass profiles, as well as the virial values of the mass and the radius, are consistent among the different methods, and they agree with the results coming from the X-ray (Akamatsu et al. 2011) and the weak lensing (Umetsu et al. 2009) analyses. Serra et al. (2011) find that the caustic technique tends to overestimate the value of mass in the central region of a cluster. Our results appear consistent with this finding, because the caustic mass profile increases more rapidly with radius in the inner part with respect to the profiles coming from DK and MAMPOSSt.

The parameters describing the mass profile are then used to invert the Jeans equation and compute the velocity anisotropy for the three different samples considered. Despite large uncertainties, the $\beta(r)$ profile for the full set of cluster members is compatible with isotropy, becoming weakly radially anisotropic in the outer regions. The behaviour of the RED sample is different. Although compatible within $1 \sigma$ with isotropy at all radii within $r_{200}$, it is suggestive of a decreasing slope, starting slightly radially anisotropic in the centre and becoming slightly tangentially anisotropic at large radii. The difference between the $\beta(r)$ profiles for the ALL sample and the RED sample is mainly due to the behaviour of the BLUE sample, which shows radial anisotropy at all radii except in the centre where it is isotropic.

With the information obtained on A2142, we were able to test some theoretical relations regarding the interplay between the mass distribution and the internal kinematics of a cluster. We investigated the radial profile of the pseudo phase-space density (PPSD) $Q(r)$, as well as its radial counterpart $Q_{r}(r)$. When we considered the total density profile to compute $Q$ and $Q_{r}$, we found that the profiles for A2142 are weakly consistent 
with the theoretical expectations (Dehnen \& McLaughlin 2005; Ludlow et al. 2010) when considering the ALL sample, but a good agreement is observed in the RED sample. This strengthens the scenario of blue galaxies being a population of galaxies recently fallen into clusters, which have had no time to reach an equilibrium configuration yet, or are heavily contaminated by interlopers.

We estimated the PPSD profile of the total matter, making the assumption that the galaxy velocity dispersion is a good proxy for the total matter dynamics. When we replace the total mass density by the number density of the tracer for which we compute the velocity dispersion, the PPSDs are shallower power laws than those found by Dehnen \& McLaughlin (2005) in simulated $\Lambda \mathrm{CDM}$ haloes.

The velocity anisotropy configuration of the internal kinematics reflects the formation history of the cluster. Therefore we expect a relation between the velocity anisotropy and the potential of the cluster. A relation linking the $\beta(r)$ profile and $\gamma(r)$, the logarithmic slope of the potential, has been analysed and compared to the theoretical results provided by Hansen \& Moore (2006), resulting in weak agreement. A correlation between the $\beta$ and $\gamma$ appears to hold out to $\gamma \simeq-2.3$ in the RED sample, corresponding to a radial distance $\simeq 0.5 r_{200} \simeq 1 \mathrm{Mpc}$. Interestingly, cluster-mass simulated $\Lambda \mathrm{CDM}$ haloes also follow the Hansen \& Moore relation out to slopes of $\gamma \approx-2.3$ but not beyond (see Fig. 17 of Lemze et al. 2012). Our considerations do not change when we compute the $\beta-\gamma$ relation using the logarithmic slope of the number density profile of galaxies instead of the total matter density profile.

Before reaching any conclusion, we must keep in mind that the present theoretical studies of the $\beta-\gamma$ and PPSD relations lack the influence of baryonic physics, as well as the dynamical processes acting on galaxies but not on DM particles. This might induce the differences when comparing the theoretical predictions with the observational results.

When we have better control of these properties, the PPSD might provide a powerful tool for the study of structure formation. As an example, the PPSD of the blue galaxies in A2142 appears very different from what has been found for the blue galaxies in another cluster, MACS J1206.2-0847 at $z=0.44$ (Biviano et al. 2013). This discrepancy suggests interesting perspectives for understanding the formation of galaxy clusters.

Acknowledgements. We thank Luca Ciotti, Colin Norman, Barbara Sartoris, and Radek Wojtak for useful discussions, an anonymous referee for helpful comments, and Anthony Lewis for building the public CosmoMC Markov Chain Monte Carlo code. We acknowledge partial support from "Consorzio per la Fisica - Trieste" and from MIUR PRIN2010-2011 (J91J12000450001). A.B. and E.M. acknowledge the hospitality of the Institut d'Astrophysique de Paris.

\section{References}

Akamatsu, H., Hoshino, A., Ishisaki, Y., et al. 2011, PASJ, 63, 1019 Bartelmann, M. 1996, A\&A, 313, 697 Beers, T. C., Flynn, K., \& Gebhardt, K. 1990, AJ, 100, 32

Bhattacharya, S., Habib, S., Heitmann, K., \& Vikhlinin, A. 2013, ApJ, 766, 32 Binney, J., \& Mamon, G. A. 1982, MNRAS, 200, 361

Binney, J., \& Tremaine, S. 1987, Galactic dynamics (Princeton: Princeton University Press)

Biviano, A., \& Girardi, M. 2003, ApJ, 585, 205

Biviano, A., \& Katgert, P. 2004, A\&A, 424, 779
Biviano, A., \& Poggianti, B. M. 2009, A\&A, 501, 419

Biviano, A., et al. 2013, A\&A, 558, A1

Böhringer, H., \& Werner, N. 2010, A\&ARv, 18, 127

Burkert, A. 1995, ApJ, 447, L25

Ciotti, L., \& Morganti, L. 2010, MNRAS, 408, 1070

Collister, A. A., \& Lahav, O. 2005, MNRAS, 361, 415

De Boni, C., Ettori, S., Dolag, K., \& Moscardini, L. 2013, MNRAS, 428, 2921

De Grandi, S., \& Molendi, S. 2002, ApJ, 567, 163

Dehnen, W., \& McLaughlin, D. E. 2005, MNRAS, 363, 1057

Dejonghe, H., \& Merritt, D. 1992, ApJ, 391, 531

den Hartog, R., \& Katgert, P. 1996, MNRAS, 279, 349

Diaferio, A. 1999, MNRAS, 309, 610

Diaferio, A., \& Geller, M. J. 1997, ApJ, 481, 633

Ettori, S., De Grandi, S., \& Molendi, S. 2002, A\&A, 391, 841

Falco, M., Mamon, G. A., Wojtak, R., Hansen, S. H., \& Gottlöber, S. 2013, MNRAS, 436, 2639

Gebhardt, K., Pryor, C., Williams, T. B., \& Hesser, J. E. 1994, AJ, 107, 2067

Geller, M. J., Diaferio, A., \& Kurtz, M. J. 1999, ApJ, 517, L23

Geller, M. J., Diaferio, A., Rines, K. J., \& Serra, A. L. 2013, ApJ, 764, 58

Gifford, D., Miller, C., \& Kern, N. 2013, ApJ, 773, 116

Hansen, S. H., \& Moore, B. 2006, New Astron., 11, 333

Hernquist, L. 1990, ApJ, 356, 359

Jeans, J. H. 1904, The Dynamical Theory of Gases (Cambridge University Press) http: //archive.org/stream/dynamical theory@1 jeangoog, chap. 7

Katgert, P., Biviano, A., \& Mazure, A. 2004, ApJ, 600, 657

Lemze, D., Wagner, R., Rephaeli, Y., et al. 2012, ApJ, 752, 141

Lewis, A., \& Bridle, S. 2002, Phys. Rev. D, 66, 103511

Łokas, E. L. 2002, MNRAS, 333, 697

Łokas, E. L., \& Mamon, G. A. 2001, MNRAS, 321, 155

Łokas, E. L., \& Mamon, G. A. 2003, MNRAS, 343, 401

Ludlow, A. D., Navarro, J. F., Springel, V., et al. 2010, MNRAS, 406, 137

Ludlow, A. D., Navarro, J. F., White, S. D. M., et al. 2011, MNRAS, 415, 3895

Ludlow, A. D., Navarro, J. F., Boylan-Kolchin, M., et al. 2013, MNRAS, 432, 1103

Mahajan, S., Mamon, G. A., \& Raychaudhury, S. 2011, MNRAS, 416, 2882

Mamon, G. A. 1987, ApJ, 321, 622

Mamon, G. A., \& Łokas, E. L. 2005a, MNRAS, 362, 95

Mamon, G. A., \& Łokas, E. L. 2005b, MNRAS, 363, 705

Mamon, G. A., Łokas, E., Dekel, A., Stoehr, F., \& Cox, T. J. 2006, in EAS Pub. Ser. 20, eds. G. A. Mamon, F. Combes, C. Deffayet, \& B. Fort, 139

Mamon, G. A., Biviano, A., \& Murante, G. 2010, A\&A, 520, A30

Mamon, G. A., Biviano, A., \& Boué, G. 2013, MNRAS, 429, 3079

Markevitch, M., Ponman, T. J., Nulsen, P. E. J., et al. 2000, ApJ, 541, 542

Moss, C., \& Dickens, R. J. 1977, MNRAS, 178, 701

Munari, E., Biviano, A., Borgani, S., Murante, G., \& Fabjan, D. 2013, MNRAS, 430, 2638

Navarro, J. F., Frenk, C. S., \& White, S. D. M. 1996, ApJ, 462, 563

Navarro, J. F., Frenk, C. S., \& White, S. D. M. 1997, ApJ, 490, 493

Navarro, J. F., Hayashi, E., Power, C., et al. 2004, MNRAS, 349, 1039

Okabe, N., \& Umetsu, K. 2008, PASJ, 60, 345

Owers, M. S., Nulsen, P. E. J., \& Couch, W. J. 2011, ApJ, 741, 122

Press, W. H., Teukolsky, S. A., Vetterling, W. T., et al. 1993, The Observatory, 113,214

Richardson, T., \& Fairbairn, M. 2013, MNRAS, 432, 3361

Rossetti, M., Eckert, D., De Grandi, S., et al. 2013, A\&A, 556, A44

Sanchis, T., Łokas, E. L., \& Mamon, G. A. 2004, MNRAS, 347, 1198

Serra, A. L., \& Diaferio, A. 2013, ApJ, 768, 116

Serra, A. L., Diaferio, A., Murante, G., \& Borgani, S. 2011, MNRAS, 412, 800

Solanes, J. M., \& Salvador-Solé, E. 1990, A\&A, 234, 93

Taylor, J. E., \& Navarro, J. F. 2001, ApJ, 563, 483

Tiret, O., Combes, F., Angus, G. W., Famaey, B., \& Zhao, H. S. 2007, A\&A, 476, L1

Umetsu, K., Birkinshaw, M., Liu, G.-C., et al. 2009, ApJ, 694, 1643

van den Bosch, F. C. 2002, MNRAS, 331, 98

van der Marel, R. P., Magorrian, J., Carlberg, R. G., Yee, H. K. C., \& Ellingson, E. 2000, AJ, 119, 2038

Van Hese, E., Baes, M., \& Dejonghe, H. 2011, ApJ, 726, 80

Vogelsberger, M., Mohayaee, R., \& White, S. D. M. 2011, MNRAS, 414, 3044

Wojtak, R., \& Łokas, E. L. 2010, MNRAS, 408, 2442

Wu, H.-Y., Hahn, O., Evrard, A. E., Wechsler, R. H., \& Dolag, K. 2013, MNRAS, 436, 460 